\title{
A prospective study of clinical characteristics and outcomes of acute kidney injury in a tertiary care Centre
}

\author{
Su Hooi Teo ${ }^{1 *}$, Kian-Guan Lee ${ }^{1}$, Riece Koniman', Alvin Ren Kwang Tng ${ }^{1}$, Zhong Hong Liew ${ }^{1}$, Thin Thiri Naing ${ }^{2}$, \\ Huihua Li ${ }^{2,3}$, Ru Yu Tan ${ }^{1}$, Han Khim Tan', Hui Lin Choong ${ }^{1}$, W. Y. Marjorie Foo ${ }^{1}$ and Manish Kaushik ${ }^{1}$
}

\begin{abstract}
Background: Acute kidney injury (AKI) is a major global health problem. We aim to evaluate the epidemiology, risk factors and outcomes of AKI episodes in our single centre.

Methodology: We prospectively identified $422 \mathrm{AKI}$ and acute on chronic kidney disease episodes in 404 patients meeting KDIGO definitions using electronic medical records and clinical data from 15th July to 22nd October 2016, excluding patients with baseline estimated GFR (eGFR) of $<15 \mathrm{~mL} / \mathrm{min}$. Patients were followed up till 6 months after AKI diagnosis.

Results: The mean age was $65.8 \pm 14.1$. Majority of patients were male (58.2\%) of Chinese ethnicity (68.8\%). One hundred and thirty-two patients (32.6\%) were diagnosed in acute care units. Seventy-five percent of patients developed AKI during admission in a non-Renal specialty. Mean baseline eGFR was $50.2 \pm 27.7 \mathrm{~mL} / \mathrm{min}$. Mean creatinine at AKI diagnosis was $297 \pm 161 \mu \mathrm{mol} / \mathrm{L}$. Renal consultations were initiated at KDIGO Stages 1, 2 and 3 in 58.9, 24.5 and 16.6\% of patients, respectively. Three hundred and ten (76.7\%) patients had a single etiology of AKI with the 3 most common etiologies of AKI being pre-renal (27.7\%), sepsis-associated (25.5\%) and ischemic acute tubular necrosis (15.3\%). One hundred and nine (27\%) patients received acute renal replacement therapy. In-hospital mortality was $20.3 \%$. Six-month mortality post-AKI event was 9.4\%. On survival analysis, patients with KDIGO Stage 3 AKI had significantly shorter survival than other stages.

Conclusion: AKI is associated with significant in-hospital to 6-month mortality. This signifies the pressing need for AKI prevention, early detection and intervention in mitigating reversible risk factors in order to optimize clinical outcomes.
\end{abstract}

Keywords: Acute kidney injury, Dialysis, Mortality

\section{Background}

Acute kidney injury is one of the major complications in acutely ill patients and imposes significant mortality and morbidity globally [1-5]. AKI may be present on admission to hospital or develop during the course of hospitalization [6]. Based on the Kidney Disease, Improving Global Outcomes (KDIGO) report, the incidence of AKI in hospitalized patients ranges from 17 to 31\% [7-9]. AKI-related inpatient care is also associated with increased healthcare costs due to prolonged hospitalizations, additional investigations and the development of complications such as the need for renal replacement therapy (RRT), cardiovascular

\footnotetext{
* Correspondence: teo.su.hooi@singhealth.com.sg

1 Department of Renal Medicine, Academia, Singapore General Hospital, 20 College Road, Singapore 169856, Singapore

Full list of author information is available at the end of the article
}

complications and re-admissions [10-12]. The $2009 \mathrm{Na}-$ tional Confidential Enquiry into Patient Outcomes and Death (NCEPOD) reported that $50 \%$ of patients who died from AKI received suboptimal care and $14 \%$ of AKI was avoidable [13]. In 2013, the International Society of Nephrology launched the 0by25 initiative of improving timely diagnosis and treatment of AKI globally with an aim to eliminate preventable deaths from AKI worldwide by 2025 [8]. In view of this, a considerably greater attention has been paid to Asian countries. The risk factors, myriad of etiologies and consequences of AKI have been well-delineated $[6,14,15]$. Given the diversity in culture, ethnicity, climate and socioeconomic status, it is not surprising that a difference in etiology, incidence and risk factors of AKI exists in various parts of Asia. The pooled- incidences of AKI in hospitalized patients in Asia vary from $9.0 \%$ in

(c) The Author(s). 2019 Open Access This article is distributed under the terms of the Creative Commons Attribution 4.0 International License (http://creativecommons.org/licenses/by/4.0/), which permits unrestricted use, distribution, and 
Central Asia to 31.0\% in Southeastern Asia [7]. The development of AKI has been shown to progress to chronic kidney disease (CKD) and end stage renal disease (ESRD). To date, the data on acute kidney injury in Singapore is scattered. Therefore, we aimed to (i) analyze the distribution of AKI in different clinical units of adults admitted to a hospital in Singapore, and (ii) describe the clinical characteristics, risk profiles and outcomes of AKI.

\section{Methods}

\section{Study population}

Data on patients referred to the Nephrology Department in Singapore General Hospital (a 1785-bedded tertiary hospital) and diagnosed with AKI by KDIGO (2012) criteria from 15th July to 22nd October 2016 were prospectively collected from electronic medical records and clinical notes. Patients with estimated glomerular filtration rate (eGFR) of $\leq 15 \mathrm{~mL} / \mathrm{min}$ were excluded. The study protocol was approved by the SingHealth Centralized Institutional Review Board (IRB). Patients were followed up till 6 months after AKI diagnosis.

\section{Definition}

We studied AKI according to the KDIGO 2012 AKI criteria, (i) increase in serum creatinine $\geq 26.5 \mu \mathrm{mol} / \mathrm{L}$ within $48 \mathrm{~h}$, (ii) increase in serum creatinine $\geq 1.5 \mathrm{x}$ from baseline serum creatinine within the prior 7 days. Baseline serum creatinine was defined as the result on admission or the latest available serum creatinine within the preceding 12 months prior to admission, whichever available. Patients were included if serum creatinine fulfilled criteria for minimum KDIGO 2012 stage within $24 \mathrm{~h}$ of admission. Hypotension preceding diagnosis of AKI was defined as mean arterial pressure (MAP) of less than $70 \mathrm{mmHg}$ or the use of inotropes or vasopressors.

\section{Statistical analysis}

Mean and standard deviation (SD) were reported for continuous variables, while frequency and proportion were reported for categorical data. Overall survival was defined from the date of AKI diagnosis to the date of death, or last follow-up date for censored cases. Overall survival was estimated by the Kaplan-Meier method. Log-rank test was used to compare survival curves. Univariable Cox regression was carried out to evaluate the effects of potential factors on overall survival. All the variables with $p$-value of $<0.2$ by univariable Cox regression were included in the multivariable analysis. Multivariable model was built up by means of reduced model selection using Akaike's information criterion (AIC). For all analyses, $p$ value is taken as statistically significant when it is $<0.05$. R 3.4.2 (https:// www.r-project.org) was used for analysis.

\section{Results}

\section{Clinical characteristics of AKI patients}

A total of 422 episodes of AKI in 404 patients were identified to have AKI. The clinical characteristics of the patients are shown in Table 1 . The severity of AKI was classified as KDIGO AKI Stage 1 in 238 (58.9\%) patients; Stage 2 in 99 (24.5\%) patients and Stage 3 in 67 (16.6\%) patients. Among critically ill patients, $89(22 \%)$ patients with AKI were detected whilst in Intensive Care Unit (ICU) and 43 (10.6\%) in Intermediate Care Area (ICA) or High Dependency Units. Two hundred seventy-two (67.3\%) patients developed AKI in the general wards, of which $67 \%$ were in Stage 1. In our study, $147(36.1 \%)$ patients with AKI were detected in the medical departments, while $81(20 \%)$ were detected in the cardiac units and 78 (19.3\%) in the surgical departments. Ninety-eight (24.3\%) patients with AKI were identified in the nephrology unit.

Of the 404 patients, 235 (58.2\%) were male and 169 (41.8\%) were female. The mean age was $65.8 \pm 14.1$ years, with $58.9 \%$ older than 65 years. The ethnicity distribution reflected that of our hospital population as a whole. Mean baseline serum creatinine was $150 \pm 71 \mu \mathrm{mol} / \mathrm{L}$, with a corresponding eGFR of $50 \pm 27.7 \mathrm{~mL} / \mathrm{min}$. With regards to comorbidities, 302 (74.8\%) had hypertension, 230 (56.9\%) had diabetes mellitus, and 187 (46.3\%) had ischemic heart disease. Seventy-eight cases (19.3\%) of AKI occurred in the background of underlying malignancy. Mean serum creatinine at AKI diagnosis was $297.5 \pm 160.7 \mu \mathrm{mol} / \mathrm{L}$. The most frequent cause of AKI was noted to be pre-renal cause, with an occurrence in 112 (27.7\%) patients, followed by sepsis-associated AKI and ischemic acute tubular necrosis (ATN) occurring in 105 (26\%) and 62 (15.3\%) patients, respectively. Dialysis was carried out in 109 (27\%) of our AKI patients, of which $62(56.9 \%)$ received continuous renal replacement therapy (CRRT).

\section{Univariable analysis}

The univariate analysis of risk factors associated with mortality for patients with AKI is shown in Table 2. Elderly patients had a statistically significant shorter survival (HR $1.54,95 \% 1.07-2.22, p=0.0201$ ). Malay ethnicity was noted to have a lower risk of mortality (HR 0.55 , 95\% CI $0.33-$ $0.94, p=0.0272$ ). Baseline eGFR of $>60 \mathrm{~mL} / \mathrm{min}$ was associated with a higher risk of mortality (HR 1.54, 95\% CI 1.08-2.21, $p=0.0180$ ). Renal transplant recipients had a lower risk of death (HR 0.11, 95\% CI 0.03-0.46, $p=$ 0.0023). Paradoxically, hypertension was found to be associated with a lower mortality in AKI (HR 0.5, 95\% CI $0.35-$ $0.72, p=0.0002)$. Multifactorial AKI was also found to be associated with higher mortality (HR 1.81, 95\% CI 1.25$2.61, p=0.0017)$. Patients with hypernatremia had a lower survival (HR 2.31, 95\% CI 1.21-4.38, $\mathrm{p}=0.0180$ ). Presence of hypotension in the preceding $48 \mathrm{~h}$ prior to occurrence of AKI was strongly associated with mortality (HR 3.35, 
Table 1 Baseline Characteristics of the study cohort (per patient, total no $=404$ )

\begin{tabular}{|c|c|}
\hline Characteristics & Mean (SD) \\
\hline$\overline{\text { Age }}$ & $65.8(14.1)$ \\
\hline BMI & $24.7(5.3)$ \\
\hline Baseline creatinine & $150 \mu \mathrm{mol} / \mathrm{L}(71)$ \\
\hline Baseline eGFR & $50.2 \mathrm{~mL} / \mathrm{min}(27.7)$ \\
\hline Creatinine at AKI diagnosis & $297.5 \mu \mathrm{mol} / \mathrm{L}(160.7)$ \\
\hline \multicolumn{2}{|l|}{ Gender (\%) } \\
\hline Male & $235(58.2)$ \\
\hline Female & $169(41.8)$ \\
\hline \multicolumn{2}{|l|}{ Ethnicity (\%) } \\
\hline Chinese & $278(68.8)$ \\
\hline Malay & $71(17.6)$ \\
\hline Indian & $39(9.7)$ \\
\hline Others & $16(4)$ \\
\hline \multicolumn{2}{|l|}{ Primary Etiology of AKI } \\
\hline Pre-renal & $112(27.7)$ \\
\hline Ischemic acute tubular necrosis & $62(15.3)$ \\
\hline Cardio-renal syndrome & $33(8.2)$ \\
\hline Hypertensive emergency & $18(4.5)$ \\
\hline Allograft rejection & $1(0.2)$ \\
\hline Abdominal compartment syndrome & $2(0.5)$ \\
\hline Tubular obstruction & $9(2.2)$ \\
\hline Drug- associated AKI & $12(3)$ \\
\hline Cardiac surgery associated-AKI & $20(5)$ \\
\hline Sepsis associated-AKI & $105(26)$ \\
\hline Contrast-induced AKI (CIN) & $7(1.7)$ \\
\hline Obstructive uropathy & $9(2.2)$ \\
\hline Glomerulonephritis & $10(2.5)$ \\
\hline Hepatorenal syndrome & $4(1)$ \\
\hline \multicolumn{2}{|l|}{ Single or multiple etiology } \\
\hline Single & $310(76.7)$ \\
\hline Multiple & $94(23.3)$ \\
\hline \multicolumn{2}{|l|}{ Location } \\
\hline General wards & $272(67.3)$ \\
\hline Intensive care area/ High dependency unit & $43(10.6)$ \\
\hline Intensive care unit & $89(22)$ \\
\hline Characteristics & Frequency (\%) \\
\hline \multicolumn{2}{|l|}{ Service where patient is located } \\
\hline Cardiac & $81(20)$ \\
\hline Surgery & $78(19.3)$ \\
\hline Medical & $147(36.1)$ \\
\hline Renal & $98(24.3)$ \\
\hline \multicolumn{2}{|l|}{ Comorbidities } \\
\hline Diabetes Mellitus & $230(56.9)$ \\
\hline
\end{tabular}

Table 1 Baseline Characteristics of the study cohort (per patient, total no = 404) (Continued)

\begin{tabular}{ll}
\hline Characteristics & Mean (SD) \\
\hline Hypertension & $302(74.8)$ \\
Cardiovascular disease & $187(46.3)$ \\
Atrial fibrillation & $58(14.4)$ \\
Cerebrovascular accident & $57(14.1)$ \\
Cancer & $78(19.3)$ \\
KDIGO stage at AKI diagnosis & \\
1 & $238(58.9)$ \\
2 & $99(24.5)$ \\
3 & $67(16.6)$ \\
\hline
\end{tabular}

95\% 2.18-5.13, $p<0.0001)$. There was almost a twofold increased risk of mortality in AKI Stage 3 (HR 1.88, 95\% CI $1.23-2.88, p=0.0034)$. AKI patients who required renal replacement therapy had a significantly higher mortality (HR 2.74, 95\% CI 1.93-3.89, $p<0.001$ ).

\section{Multivariable analysis}

In the multivariable analysis shown in Table 3, the independent baseline variable that was significantly associated with mortality was age more than 65 (HR 1.46, 95\% CI 1.00-2.13, $p=0.0483)$. Interestingly, AKI in the setting of renal transplant recipients (HR 0.17, 95\% CI 0.04-0.70, $p=0.0143$ ) and hypertension (HR $0.53,95 \%$ CI $0.37-0.78, p=0.0010$ ) were significantly associated with a lower risk of mortality. Presence of hypotension in the preceding $48 \mathrm{~h}$ (HR $2.57,95 \% \mathrm{CI}$ 1.63-4.07, $p=0.0001$ ) prior to development of AKI and AKI requiring dialysis (HR 1.67, 95\% CI 1.14-2.44, $p=0.0084$ ) were significantly associated with death.

Risk factors associated with mortality based on one episode of AKI per patient are presented in the Additional file 1: Table S1 and Table S2.

\section{Outcomes of AKI}

The outcomes associated with AKI are shown in Table 4. Median serum creatinine at discharge was $176 \mu \mathrm{mol} / \mathrm{L}$ (IQR 114-278 $\mu \mathrm{mol} / \mathrm{L}$ ). Upon discharge, 16 (4\%) patients still required dialysis support. In-hospital mortality rate was $20.3 \%$ while 6 -month mortality was $9.4 \%$.

The survival rates at 3- and 6-months post-AKI diagnosis according to severity of AKI, are shown in Table 5 . AKI was associated with decreased survival according to severity of AKI stages at 3 months post-AKI episode (75.9, 95\% CI 70.7-81.5\% for Stage 1; 73.7, 95\% CI 65.3-83.1\% for Stage 2, and 57.4, 95\% CI $46.7-70.4 \%$ for Stage 3). There were significant differences in survival between the severity of AKI stages (log-rank test $p$-value 0.0091).

At 6 months, AKI was associated with decreased survival with worsening severity of AKI stages: $71.4 \%$ (95\% CI 65.9-77.3\%) for Stage 1, 70.5\% (95\% CI 61.9- 
Table 2 Univariate Analysis of Risk Factors associated with Mortality for Patients with AKI

\begin{tabular}{l}
\hline Age \\
$\leq 65$ \\
$>65$ \\
BMl \\
$\quad \leq 30$ \\
$>30$ \\
Baseline eGFR (mL/min) \\
$\quad \leq 60$ \\
$>60$ \\
Urea at RRT Initiation (mmol/L) \\
$\quad \leq 30$ \\
$>30$ \\
Sodium at RRT Initiation (mmol/L) \\
$\leq 146$ \\
$>146$
\end{tabular}

Serum potassium at RRT Initiation $(\mathrm{mmol} / \mathrm{L})$

$$
\leq 5
$$$$
>5
$$

Serum chloride at RRT Initiation $(\mathrm{mmol} / \mathrm{L})$

$$
\begin{aligned}
& \leq 107 \\
& >107
\end{aligned}
$$

Serum bicarbonate at RRT Initiation (mmol/L)

$$
\leq 19
$$$$
>19
$$

Serum albumin at RRT Initiation $(\mathrm{g} / \mathrm{dL})$

$$
\leq 40
$$$$
>40
$$

Hemoglobin at RRT Initiation ( $\mathrm{g} / \mathrm{dL}$ )

$$
\begin{aligned}
& \leq 10 \\
& >10
\end{aligned}
$$

$$
\operatorname{HR}(95 \% \mathrm{Cl})
$$

Reference

$$
1.54(1.07,2.22)
$$

Reference

$0.97(0.57,1.65)$

0.9138

Reference

$1.54(1.08,2.21)$

0.0180

Reference

$0.82(0.48,1.39)$

0.4533

Reference

$2.31(1.21,4.38)$

0.0108

Reference

$0.9(0.51,1.58)$

0.7028

Reference

$1.02(0.59,1.77)$

0.9327

Reference

$1.15(0.69,1.94)$

0.5847

Reference

$5.03(0.67,37.6)$

0.1153

Reference

$0.74(0.43,1.28)$

Serum lactate at RRT Initiation ( $\mathrm{mmol} / \mathrm{L}$ )

$$
\leq 2.2
$$$$
>2.2
$$

Gender

Male

Female

Ethnicity

Chinese
Malay
Indian
Others

\section{Reference}

$1.75(0.98,3.13)$

Reference

$1.12(0.79,1.58)$

Reference

$0.55(0.33,0.94)$

0.0272

$0.52(0.25,1.07) \quad 0.0775$

$0.71(0.26,1.94) \quad 0.5059$
Table 2 Univariate Analysis of Risk Factors associated with

\begin{tabular}{|c|c|}
\hline No & Reference \\
\hline Ye & $1.4(0.89,2.2)$ \\
\hline
\end{tabular}
Mortality for Patients with AKI (Continued)

\begin{tabular}{lll}
\hline & $H R(95 \% \mathrm{Cl})$ & $P$ value \\
\hline Renal transplant & HR $(95 \% \mathrm{Cl})$ & $P$ value \\
No & & \\
Yes & Reference & \\
Diabetes mellitus & $0.11(0.03,0.46)$ & 0.0023 \\
No & & \\
Yes & Reference & \\
& $0.83(0.59,1.18)$ & 0.3062
\end{tabular}

Hypertension

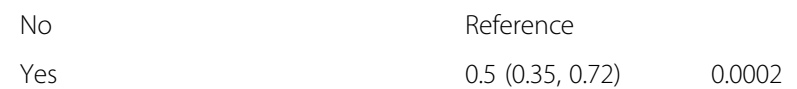

Cardiovascular disease

No Reference

Yes

$1.22(0.87,1.73) \quad 0.2538$

Atrial fibrillation

Cerebrovascular accident

No Reference

$\begin{array}{lll}\text { Yes } & 0.95(0.58,1.57) & 0.8555\end{array}$

Cancer

No Reference

$\begin{array}{lll}\text { Yes } & 1.29(0.85,1.93) & 0.2294\end{array}$

Any hypotension in the preceding $48 \mathrm{~h}$

No Reference

$\begin{array}{lll}\text { Yes } & 3.35(2.18,5.13)<0.0001\end{array}$

NA $\quad 1.1(0.67,1.8) \quad 0.7208$

KDIGO stage at AKI diagnosis

1 Reference

$2 \quad 1.04(0.67,1.61) \quad 0.8646$

$3 \quad 1.88(1.23,2.88) \quad 0.0034$

$0.2841 \quad$ Single or multiple etiology of AKI

$\begin{array}{lll}\text { Single } & \text { Reference } & \\ \text { Multiple } & 1.81(1.25,2.61) & 0.0017\end{array}$

Did the patient receive RRT during admission

No Reference

\begin{tabular}{lll} 
Yes & $2.74(1.93,3.89)$ & $<0.0001$ \\
\hline
\end{tabular}

$80.3 \%$ ) for Stage 2, 54.4\% (95\% CI 43.8-67.6\%) for Stage 3 (log-rank test p-value 0.0091). Fig. 1 shows the Kaplan-Meier estimated survival according to AKI stages. On survival analysis, patients with KDIGO Stage 1 and 2 AKI had significantly better survival than Stage 3 AKI. 
Table 3 Multivariate Analysis of Risk Factors associated with Mortality for Patients with AKI

\begin{tabular}{lll}
\hline & HR $(95 \% \mathrm{Cl})$ & P value \\
\hline $\begin{array}{l}\text { Age } \\
\leq 65\end{array}$ & Reference & \\
$>65$ & $1.46(1.00,2.13)$ & 0.0483 \\
Renal Transplant & & \\
No & Reference & 0.0143 \\
Yes & $0.17(0.04,0.70)$ & \\
Hypertension & & \\
$\quad$ No & Reference & \\
Yes & $0.53(0.37,0.78)$ & \\
Any hypotension in the & & \\
preceding 48h & & 0.0010 \\
No & Reference & \\
Yes & $2.57(1.63,4.07)$ & 0.1375 \\
NA & $1.47(0.88,2.44)$ & \\
Did the patient receive & & 0.0084 \\
RRT during admission & & \\
No & Reference & \\
Yes & $1.67(1.14,2.44)$ & \\
\hline
\end{tabular}

\section{Discussion}

This study investigated a cohort of 404 patients admitted to a tertiary hospital over 100 days. Patients with AKI were predominantly identified in non-nephrology specialties, and nephrologists' involvement started after consultation was requested. Sixty percent $(60 \%)$ of referrals for AKI were at Stage 1 AKI at point of nephrology consult. We have demonstrated that AKI developed in $22 \%$ of critically ill patients. This is consistent with other studies showing incidence rate of AKI during ICU stays varying from 22 to 67\% [16-19]. The epidemiological data, clinical features and etiology on AKI in Asian countries differ from that of what we found in our data, especially with regards to precipitants of AKI. To-date, there is a paucity of data from

Table 4 Clinical outcomes of patients with AKI

\begin{tabular}{ll}
\hline Outcomes & Patients $(n=404)(\%)$ \\
\hline Total in-hospital mortality & $82(20.3)$ \\
Received RRT during admission & $109(27)$ \\
Required dialysis support upon discharge & $16(4)$ \\
$\begin{array}{l}\text { Median serum creatinine on discharge } \\
(\mu \mathrm{mol} / \mathrm{L})\end{array}$ & $176(\mathrm{IQR}$ 114-278 \\
$\begin{array}{l}\text { Initial modality } \\
\text { Intermittent hemodialysis }\end{array}$ & $6(1.5)$ \\
$\quad$ Sustained low efficiency dialysis (SLED) & $41(10.1)$ \\
$\quad$ Continuous renal replacement therapy (CRRT) & $62(15.3)$ \\
6-month mortality & $38(9.4)$ \\
\hline
\end{tabular}

Table 5 Survival rate 3 and 6 months post-AKI diagnosis according to severity of AKI

\begin{tabular}{lll}
\hline & \multicolumn{2}{l}{ Survival rate $(95 \% \mathrm{Cl})$} \\
\cline { 2 - 3 } KDIGO stage & 3 months survival & 6 months survival \\
\hline 1 & $75.9 \%(70.7,81.5 \%)$ & $71.4 \%(65.9,77.3 \%)$ \\
2 & $73.7 \%(65.3,83.1 \%)$ & $70.5 \%(61.9,80.3 \%)$ \\
3 & $57.4 \%(46.7,70.4 \%)$ & $54.4 \%(43.8,67.6 \%)$ \\
\hline
\end{tabular}

Singapore on clinical characteristics, etiologies and outcomes of patients with AKI. Our study was intended to give us an insight into this. Locally, Chua et al. evaluated 207 patients with septic AKI and mortality in Singapore and reported that a 1 -year mortality rate of $40 \%$, with high daily fluid balance and frusemide administration being modifiable risk factors [20]. In terms of cardiac surgery-associated AKI in South-East Asian population, Chew et al. reported that $35.3 \%$ of patients developed AKI after cardiac surgery, with Indian and Malay ethnicity having a higher risk than Chinese ethnicity [21]. Our study provides novel facts on the major affected clinical settings of AKI, clinical characteristics, risk factors and outcomes associated with AKI across different etiological insults.

We identified pre-renal cause as a precipitant of AKI in $27.7 \%$, a lower proportion compared to a study by Tang et al. reporting pre-renal cause of AKI of $49.1 \%$ from the medical departments [22]. Volume resuscitation to replace ongoing losses and restoration to baseline volume status is crucial in the event of true extracellular fluid volume depletion. Knowledge of baseline weight, careful attention to intake and output and ongoing serial weight measurement and volume status assessment directs the strategy for resuscitation [23, 24].

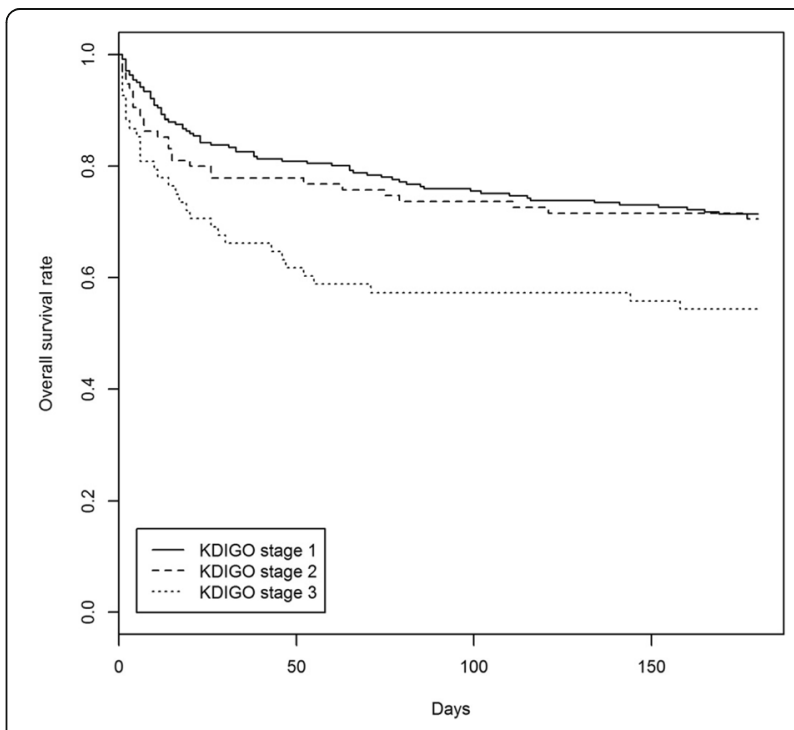

Fig. 1 Kaplan-Meier estimated survival for severity of AKI. Log-rank test $p$-value $=0.0091$ 
In our centre, $27 \%$ of AKI patients received acute renal replacement therapy (RRT), of which 56.9\% received CRRT. Our finding was relatively consistent with a study by Yang et al. reporting that $22.5 \%$ of patients received RRT [25]. The initiation of RRT in patients with severe AKI contributes to healthcare costs and is a measure of complexity of care in critically ill patients. The practice of prescription of CRRT therapy varies widely across different continents. In a retrospective analysis of 261 patients in 2 ICUs in Canada estimating the cost comparison between CRRT and intermittent hemodialysis, the weekly cost ranged from 3,486 to 5,117 Canadian dollars (depending on modality choice and anticoagulation) [26]. This cost was significantly more expensive than intermittent hemodialysis at a weekly cost of 1,342 Canadian dollars. The frequency of dialysis requirement in AKI ranges from $6.9 \%$ in Asia to $71 \%$ in the study by Beginning and Ending Supportive Therapy for the Kidney (BEST Kidney) Investigators [19] [27],

Acute kidney injury remains a common clinical problem, particularly in the elderly. Liano et al. reported a mean age of 64 years in their study involving AKI episodes occurring in adult patients admitted to any of the 13 tertiary care hospitals in Madrid [28]. The mean age of patients was 73 in two UK district hospitals reported by Meran et al [29] and 64.7 in a separate Canadian-based ICU study [30]. Similarly, patients in our study had a median age of 65.8 years. We demonstrated that patients with AKI who were 65 years old and above were associated with higher risks of mortality. Age older than 65 is not only a risk factor for impaired recovery from AKI and progression to advancedstage CKD, but the long-term survival of patients with AKI worsens with increasing age, even in non-dialysis requiring AKI [31-33]. In the setting of reduced renal reserve in the elderly [34], the higher frequency of nephrotoxins usage such as non-steroidal anti-inflammatory drugs predisposes these patients to AKI [34]. The incidence of AKI in the elderly population is expected to be on the rise given the challenges of the elderly population in the nation, along with an interplay of polypharmacy and susceptibility to nephrotoxic agents in the aged population.

In previously reported studies on transplant AKI epidemiology, the diagnosis of AKI was based on RIFLE criteria [35]. In our study, we analyzed the incidence of AKI meeting the definition in KDIGO 2012. Mehrotra et al. included 27, 232 kidney transplant recipients of which $11.3 \%$ developed AKI during the study period and it was reported that patients who developed AKI had an increased risk of death (HR 2.36, 92\% CI 2.41-2.60) [36]. However, hazard ratios for both outcomes of graft loss and death were inversely related to the severity of CKD. In our study, AKI in renal transplant recipients was associated with a lower risk of mortality paradoxically. The subgroup of the renal transplant recipients in our study was younger and had a baseline eGFR $>30 \mathrm{~mL} / \mathrm{min}$. Sepsis-associated AKI (SA-AKI) was the leading precipitant of AKI in renal transplant recipients, with the majority not requiring dialysis. Care of renal transplant recipients in our institution is provided by a dedicated renal transplant team, and any SA-AKI was aggressively managed with input from transplant infectious disease specialists. These factors may potentially contribute to a significantly lower risk of mortality in this group of AKI patients but have yet to be validated.

The identification of risk factors predicting risk of mortality is imperative so that early mitigating factors can be implemented. Many studies have attempted to identify prognostic factors in predicting AKI in critically ill patients $[37,38]$. Previous reports have demonstrated that oliguria, pre-existing CKD, AKI attributable to nephrotoxic agents, AKI severity, and multi-organ failure were significant independent risk factors for death in AKI patients. In the Madrid Acute Renal Failure Study Group by Liano et al., oliguria, sustained hypotension, assisted respiration and icterus were associated with higher mortality [28]. Interestingly in our study, an underlying comorbidity of hypertension was not significantly associated with mortality. This finding may potentially be related to the counter-effect of hypertension against ischemic insult during an episode of AKI and it remains to be validated in future studies.

Hemodynamic instability is one of the most common causes of acute kidney injury. Our understanding of kidneys receiving about $25 \%$ of our cardiac output, allows us to target adequate renal perfusion as a potential strategy to modify the risk of developing AKI. Although the mean arterial pressure (MAP) target of $\geq 65 \mathrm{mmHg}$ was defined in the Surviving Sepsis Campaign Guideline 2018, a recent study by Saito and colleagues had measured hemodynamic pressure-related parameters comparing between patients with progression of AKI versus those without AKI progression [39]. The authors measured hemodynamic pressure-related parameters including systolic arterial pressure (SAP), diastolic arterial pressure (DAP), MAP and central venous pressure (CVP), mean perfusion pressure (MPP) and diastolic perfusion pressure (DPP) and calculated deficits in the above values. The study observed a significant difference in the DPP, MPP and DAP in the patients with AKI progression, and suggested that these deficits may potentially be modifiable risk factors for the prevention of AKI progression, particularly in the patients who had undergone cardiac surgery. In our study, we found that AKI patients with hypotension in the preceding $48 \mathrm{~h}$ prior to development of AKI were reported to be strongly associated with mortality. Silva et al. reported a similar finding of hypotension being an independent risk factor for death in the intensive care units [40].

The risk of mortality with severe AKI requiring RRT remains high, particularly in the setting of critical illness, estimated to be approaching 60\% [41, 42]. Factors such as AKI stage, severity of acute non-renal organ dysfunction 
and underlying diagnosis were associated with increased risk for mortality after AKI [43]. Our study showed that 109 patients $(27 \%)$ required RRT during admission. Hsu et al. also demonstrated that the incidence of dialysis-requiring AKI had been escalating rapidly, averaging at 10\% annually in the United States with similar trends observed worldwide [44, 45]. An episode of dialysis-requiring AKI was a strong independent risk factor for long-term risk of progressive CKD and mortality [46]. Our finding of AKIrequiring dialysis as an independent predictor for mortality is consistent with previous literature [46-48].

The major strengths of our study are the detailed evaluation of the risk factors and the distribution of AKI within clinical departments. However, our findings should be interpreted in light of the following limitations. The definition of AKI used in our study was based on serum creatinine change unaccompanied by urinary output, hence leading to underestimation of the detection rate of AKI. Secondly, the study may run an inherent risk of sampling bias as it was conducted over a snapshot period. Thirdly, as the baseline serum creatinine method has not been wellunified, method such as using the minimum value of preadmission serum creatinine as a baseline creatinine has been shown to identify more patients with AKI and yield better predictive ability for 60-day mortality [49]. Multiple AKI biomarkers that are measured in the urine or plasma of patients with AKI have been discovered, including the neutrophil gelatinase-associated lipocalin (NGAL), kidney injury molecule 1 (KIM-1), liver-type fatty acid-binding protein (L-FABP), interleukin 18 (IL-18), calprotectin, urine angiotensinogen (AGT), urine microRNAs and the recently FDA-approved insulin-like growth factor-binding protein 7 $\mathrm{x}$ tissue inhibitor of metalloproteinase 2 in the USA [50]. Biomarkers for AKI diagnosis are not currently being used routinely in our local clinical practice, hence our study did not include any novel biomarkers for AKI diagnosis. In our future research, we hope to leverage the relationship of biomarkers in diagnosing AKI and predicting short and longterm outcomes of acute kidney injury in different patient care settings, given the heterogeneity of this condition. Finally, this study did not include the long-term outcomes of patient survival and the risk of ESRD after 6 months.

\section{Conclusion}

In conclusion, our study shows that AKI resulted in an in-hospital mortality of $20.3 \%$. Additionally, the AKI survivors had a mortality risk of $9.4 \%$ at 6 months. Risk factors including age above 65 , presence of hypotension in the preceding $48 \mathrm{~h}$ prior to the development of AKI and AKI requiring dialysis were significantly associated with mortality. The data we have presented will enable policies to be drawn and healthcare costs to be quantified. Thus, these findings highlight the urgent need to develop effective treatments, explore educational opportunities pertaining to AKI, and improve hospitalbased care processes aimed at early identification to prevent devastating outcomes.

\section{Additional file}

Additional file 1: Table S1. Univariable Analysis of Risk Factors associated with Mortality for Patients with AKI (based on one episode of AKI per patient). Table S2. Multivariable Analysis of Risk Factors associated with Mortality for Patients with AKI (based on one episode of AKI per patient). (DOCX $22 \mathrm{~kb}$ )

\section{Abbreviations}

AIC: Akaike's information criterion; AKI: Acute Kidney Injury; ATN: Acute tubular necrosis; Cl: Confidence interval; CKD: Chronic kidney disease; CRRT: Continuous renal replacement therapy; DAP: Diastolic arterial pressure; DPP: Diastolic perfusion pressure; eGFR: Estimated glomerular filtration rate; ESRD: End stage renal disease; HR: Hazard ratio; ICA: Intermediate Care Area; ICU: Intensive Care Unit; IRB: Institutional Review Board; KDIGO: Kidney Disease, Improving Global Outcomes; MAP: Mean arterial pressure; MPP: Mean perfusion pressure; NCEPOD: National Confidential Enquiry into Patient Outcomes and Death; RIFLE: Risk Injury, Failure, Loss of kidney function, End-stage kidney disease; RRT: Renal replacement therapy; SAAKI: Sepsis-associated acute kidney injury; SAP: Systolic arterial pressure; SD: Standard deviation

\section{Acknowledgements}

Not applicable.

\section{Authors' contributions}

MK/SHT/ CHL/ HKT/ WYMF designed the research; MKSHT/ KGL performed research; SHT/ KGL/ KR/ TA/ ZHL/ TTN collected the data; LH/ SHT/ KGL / RYT analyzed the data; All authors contributed in authorship of the manuscript.

\section{Funding}

The authors have no funding sources to declare.

\section{Availability of data and materials}

All datasets generated and/or analysed during the current study are not publicly available due to confidentiality of the data but are available from the corresponding author on reasonable request.

\section{Ethics approval and consent to participate}

The study protocol was approved by the SingHealth Centralized Institutional Review Board (IRB). The requirement for written consent was waived as no interventions or any additional investigations were required for the study purposes.

\section{Consent for publication}

Not applicable.

\section{Competing interests}

All authors have declared that they have no competing interests.

\section{Author details}

'Department of Renal Medicine, Academia, Singapore General Hospital, 20 College Road, Singapore 169856, Singapore. ${ }^{2}$ Health Services Research Unit, Bachelor of Nursing, University of Sydney, Singapore General Hospital, Singapore, Singapore. ${ }^{3}$ Centre for Quantitative Medicine, Duke-NUS Medical School, Singapore, Singapore.

Received: 12 August 2018 Accepted: 17 July 2019

Published online: 26 July 2019

\section{References}

1. Chawla LS, Eggers PW, Star RA, Kimmel PL. Acute kidney injury and chronic kidney disease as interconnected syndromes. N Engl J Med. 2014;371(1):58-66. 2. Bellomo R, Kellum JA, Ronco C. Acute kidney injury. Lancet. 2012;380(9843): 756-66. 
3. Ali T, Khan I, Simpson W, Prescott G, Townend J, Smith W, et al. Incidence and outcomes in Acute kidney injury: a comprehensive population-based study. J Am Soc Nephrol. 2007;18(4):1292-8.

4. Wald R, Quinn RR, Adhikari NK, Burns KE, Friedrich JO, Garg AX, et al. Risk of chronic Dialysis and death following Acute kidney injury. Am J Med. 2012; 125(6):585-93.

5. Hoste EAJ, Bagshaw SM, Bellomo R, Cely CM, Colman R, Cruz DN, et al. Epidemiology of acute kidney injury in critically ill patients: the multinational AKI-EPI study. Intensive Care Med. 2015;41(8):1411-23.

6. Bedford M, Stevens PE, Wheeler TWK, Farmer CKT. What is the real impact of acute kidney injury? BMC Nephrol. 2014;15(1):95

7. Susantitaphong P, Cruz DN, Cerda J, Abulfaraj M, Alqahtani F, Koulouridis I, et al. World incidence of AKl: a meta-analysis. Clin J Am Soc Nephrol. 2013;8(9):1482-93.

8. Mehta RL, Cerdá J, Burdmann EA, Tonelli M, García-García G, Jha V, et al. International Society of Nephrology's 0by25 initiative for acute kidney injury (zero preventable deaths by 2025): a human rights case for nephrology. Lancet 2015 J;385(9987):2616-2643.

9. Bouchard J, Mehta RL. Acute Kidney Injury in Western Countries. Kidney Dis (Basel, Switzerland). 2016;2(3):103-10.

10. Chertow GM, Burdick E, Honour M, Bonventre JV, Bates DW. Acute kidney injury, mortality, length of stay, and costs in hospitalized patients. J Am Soc Nephrol. 2005;16(11):3365-70.

11. Silver SA, Chertow GM. The economic consequences of Acute kidney injury. Nephron. 2017;137(4):297-301.

12. Kerr M, Bedford M, Matthews B, O'Donoghue D. The economic impact of acute kidney injury in England. Nephrol Dial Transplant. 2014;29(7):1362-8.

13. Sterwart J, Findlay G, Smith N, Kelly K, Mason M. Acute kidney injury: adding insult to injury. Natl Confid Enq into Patient Outcomes Death. 2009.

14. Mehta RL, Pascual MT, Soroko S, Savage BR, Himmelfarb J, Ikizler TA, et al. Spectrum of acute renal failure in the intensive care unit: the PICARD experience. Kidney Int. 2004;66(4):1613-21.

15. Uchino S, Kellum JA, Bellomo R, Doig GS, Morimatsu H, Morgera S, et al. Acute renal failure in critically ill patients: a multinational, multicenter study. JAMA. 2005;294(7):813-8.

16. Hoste EA, Clermont G, Kersten A, Venkataraman R, Angus DC, De Bacquer D, et al. RIFLE criteria for acute kidney injury are associated with hospital mortality in critically ill patients: a cohort analysis. Crit Care. 2006;10(3):R73.

17. Thakar CV, Christianson A, Freyberg R, Almenoff P, Render ML. Incidence and outcomes of acute kidney injury in intensive care units: a veterans administration study*. Crit Care Med. 2009;37(9):2552-8.

18. Srisawat N, Sileanu FE, Murugan R, Bellomod R, Calzavacca P, Cartin-Ceba R, et al. Variation in risk and mortality of acute kidney injury in critically ill patients: a multicenter study. Am J Nephrol. 2015;41(1):81-8.

19. Trongtrakul K, Sawawiboon C, Wang AY, Chitsomkasem A, Limphunudom $P$, Kurathong $S$, et al. Acute kidney injury in critically ill surgical patients: epidemiology, risk factors and outcomes. Nephrology (Carlton) 2017. doi: https://doi.org/10.1111/nep.13192. [Epub ahead of print].

20. Chua H-R, Wong W-K, Ong VH, Agrawal D, Vathsala A, Tay H-M, et al. Extended mortality and chronic kidney disease after septic Acute kidney injury. J Intensive Care Med 2018 Jan 1:885066618764617. doi: https://doi. org/10.1177/0885066618764617. [Epub ahead of print].

21. Chew STH, Mar WMT, Ti LK. Association of ethnicity and acute kidney injury after cardiac surgery in a south east Asian population. Br J Anaesth. 2013;110(3):397-401.

22. Tang $X$, Chen D, Yu S, Yang L, Mei C, ISN AKF 0 by 25 China Consortium Acute kidney injury burden in different clinical units: Data from nationwide survey in China. Sun J, editor. PLoS One 2017;12(2):e0171202.

23. Yamout H, Levin ML, Rosa RM, Myrie K, Westergaard S. Physician prevention of Acute kidney injury. Am J Med. 2015;128(9):1001-6.

24. Cooper CM, Fenves AZ. Before you call renal: Acute kidney injury for hospitalists. J Hosp Med. 2015;10(6):403-8.

25. Yang F, Zhang L, Wu H, Zou H, Du Y. Clinical analysis of cause, treatment and prognosis in acute kidney injury patients. Musabayane CT, editor. PLoS One 2014:9(2):e85214.

26. Manns B, Doig CJ, Lee H, Dean S, Tonelli M, Johnson D, et al. Cost of acute renal failure requiring dialysis in the intensive care unit: clinical and resource implications of renal recovery*. Crit Care Med. 2003;31(2):449-55.

27. Bagshaw SM, Uchino S, Bellomo R, Morimatsu H, Morgera S, Schetz M, et al. Septic Acute kidney injury in critically ill patients: clinical characteristics and outcomes. Clin J Am Soc Nephrol. 2007;2(3):431-9.
28. Liaño F, Pascual J. Epidemiology of acute renal failure: a prospective, multicenter, community-based study. Madrid Acute Renal Failure study group. Kidney Int. 1996;50(3):811-8.

29. Meran S, Wonnacott A, Amphlett B, Phillips A. How good are we at managing acute kidney injury in hospital? Clin Kidney J. 2014;7(2):144-50.

30. Odutayo A, Adhikari NKJ, Barton J, Burns KEA, Friedrich JO, Klein D, et al. Epidemiology of acute kidney injury in Canadian critical care units: a prospective cohort study. Can J Anesth Can d'anesthésie. 2012;59(10):934-42.

31. Anderson S, Eldadah B, Halter JB, Hazzard WR, Himmelfarb J, Horne FM, et al. Acute kidney injury in older adults. J Am Soc Nephrol. 2011;22(1):28-38.

32. Schmitt R, Coca S, Kanbay M, Tinetti ME, Cantley LG, Parikh CR. Recovery of kidney function after acute kidney injury in the elderly: a systematic review and meta-analysis. Am J Kidney Dis. 2008;52(2):262-71.

33. Cerda J, Lameire N, Eggers P, Pannu N, Uchino S, Wang H, et al. Epidemiology of Acute kidney injury. Clin J Am Soc Nephrol. 2008;3(3):881-6.

34. Musso CG, Reynaldi J, Martinez B, Pierángelo A, Vilas M, Algranati L. Renal reserve in the oldest old. Int Urol Nephrol. 2011;43(1):253-6.

35. Nakamura M, Seki G, Iwadoh K, Nakajima I, Fuchinoue S, Fujita T, et al. Acute kidney injury as defined by the RIFLE criteria is a risk factor for kidney transplant graft failure. Clin Transpl. 2012;26(4):520-8.

36. Mehrotra A, Rose C, Pannu N, Gill J, Tonelli M, Gill JS. Incidence and consequences of Acute kidney injury in kidney transplant recipients. Am J Kidney Dis. 2012;59(4):558-65.

37. Perez Valdivieso JR, Bes-Rastrollow M, Monedero P, De Irala J, La Villa FJ. Evaluation of the prognostic value of the risk, injury, failure, loss and end-stage renal failure (RIFLE) criteria for acute kidney injury. Nephrology. 2008;13(5):361-6.

38. Cruz DN, Bolgan I, Perazella MA, Bonello M, de Cal M, Corradi V, et al. North east Italian prospective hospital Renal outcome survey on Acute kidney injury (NEiPHROS-AKI): targeting the problem with the RIFLE criteria. Clin J Am Soc Nephrol. 2007;2(3):418-25.

39. Saito S, Uchino S, Takinami M, Uezono S, Bellomo R. Postoperative blood pressure deficit and acute kidney injury progression in vasopressordependent cardiovascular surgery patients. Crit Care. 2016;20(1):74.

40. da Silva Júnior GB, Daher EDF, Mota RMS, Menezes FA. Risk factors for death among critically ill patients with acute renal failure. Sao Paulo Med J. 2006; 124(5):257-63.

41. A. Molitoris B, J. Meier D, Wang E, M. Sandoval R, Sheridan E, S. Strickland J. Quantifying glomerular filtration rates: kidney function analysis method and apparatus. Recent Patents Biomarkerse 2012 Aug;2(3):209-18.

42. VA/NIH Acute Renal Failure Trial Network, Palevsky PM, Zhang JH, O'Connor TZ, Chertow GM, Crowley ST, et al. Intensity of Renal support in critically ill patients with Acute kidney injury. N Engl J Med. 2008;359(1):7-20.

43. Clec'h C, Gonzalez F, Lautrette A, Nguile-Makao M, Garrouste-Orgeas M, Jamali S, et al. Multiple-center evaluation of mortality associated with acute kidney injury in critically ill patients: a competing risks analysis. Crit Care. 2011;15(3):R128.

44. Hsu RK, McCulloch CE, Dudley RA, Lo LJ, Hsu CY. Temporal changes in incidence of Dialysis-requiring AKI. J Am Soc Nephrol. 2013;24(1):37-42.

45. Hoste EAJ, Schurgers M. Epidemiology of acute kidney injury: how big is the problem? Crit Care Med. 2008;36(4 Suppl):S146-51.

46. Lo LJ, Go AS, Chertow GM, McCulloch CE, Fan D, Ordoñez JD, et al. Dialysisrequiring acute renal failure increases the risk of progressive chronic kidney disease. Kidney Int. 2009;76(8):893-9.

47. Schiffl H, Lang SM, Fischer R. Long-term outcomes of survivors of ICU acute kidney injury requiring renal replacement therapy: a 10-year prospective cohort study. Clin Kidney J. 2012;5(4):297-302.

48. Sakhuja A, Kumar G, Gupta S, Mittal T, Taneja A, Nanchal RS. Acute kidney injury requiring Dialysis in severe Sepsis. Am J Respir Crit Care Med. 2015;192(8):951-7.

49. Thongprayoon C, Cheungpasitporn W, Kittanamongkolchai W, Srivali N, Ungprasert P, Kashani K. Optimum methodology for estimating baseline serum creatinine for the acute kidney injury classification. Nephrology. 2015; 20(12):881-6.

50. Kashani K, Cheungpasitporn W, Ronco C. Biomarkers of acute kidney injury: the pathway from discovery to clinical adoption. Clin Chem Lab Med. 2017; 55(8):1074-89.

\section{Publisher's Note}

Springer Nature remains neutral with regard to jurisdictional claims in published maps and institutional affiliations. 